\title{
The reliability of the Motor-Free Visual Perceptual Test (MVPT-3) when translated into Afrikaans, on a first language Afrikaans urban population aged 8 years 0 months to 8 years 11 months
}

\author{
Lyndsay Koch, B OT (UFS), MSc OT (Wits) \\ Junior Lecturer, Department of Occupational Therapy, School of Therapeutic Sciences, Faculty of Health Sciences, University of \\ the Witwatersrand
}

\section{Trudie Eksteen, B OT (UFS), MSc OT (Wits)}

Postgrauate Student, Department of Occupational Therapy, School of Therapeutic Sciences, Faculty of Health Sciences, University of the Witwatersrand

\section{Patricia de Witt, Dip OT (Pret), MSc OT (Wits)}

Adjunct Professor and Head, Department of Occupational Therapy, School of Therapeutic Sciences, Faculty of Health Sciences, University of the Witwatersrand

Occupational therapists and other professionals in South Africa are faced with the task of assessing and providing intervention for children from a range of cultural and language groups. The possibility of cultural variation in different cultural groups can produce confounding and inaccurate results and biased conclusions when the test is used with different groups and the results are interpreted using the norms and criteria provided by the authors. The aim of this study was to investigate the reliability of the Motor-Free Visual Perceptual Test $3^{\text {rd }}$ Edition (MPVT-3) when instructions were translated into Afrikaans. A descriptive split-half comparative research design was used to determine the internal consistency of the items on the MVPT-3 after the instructions had been translated into Afrikaans. 80 learners between the ages of 8 years 0 months and 8 years II months were selected from nine schools to participate and the translated MVPT-3 was administered once during school time. Data was analysed using Cronbach's alpha and split-half reliability statistics. Results indicated that although overall reliability was adequate, the split-half reliability revealed that the reliability scores were not adequate for half of the test, with the second half of the test showing poor reliability. There were also significant differences between boys' and girls' performance on the test, with reliability scores for boys being well below the accepted level. Thus the reliability of the MVPT-3 both within the South African population as well as when translated is questionable and the test should be interpreted with caution.

Key words: MVPT-3, psychometrics, translation

\section{INTRODUCTION}

Occupational therapists and other professionals in South Africa are faced with the task of assessing and providing intervention for children from a range of cultural and language groups. The provision of equitable therapy services to all the children of South Africa requires accurate and culturally sensitive assessment tools in order to ensure diagnostic accuracy and appropriate treatment outcomes ${ }^{1,2}$.

Within occupational therapy, psychometric standardised tests are routinely used to assess perceptual, cognitive and motor skill dysfunction in children. These tests were developed in Western countries like the United Kingdom, United States of America or Canada and were standardised on these populations with verbal instructions in English ${ }^{3}$. The use of these tests on populations outside of those on which the tests have been standardised has raised a number of concerns ${ }^{1,2}$. The possibility of cultural variation in different cultural groups and the influence of context can produce confounding and inaccurate results and biased conclusions when these tests are used with different groups and the results are interpreted using the norms and criteria provided by the authors ${ }^{4,5,6}$.

Therefore these tests are not necessarily considered reliable measures for all children in this country ${ }^{7}$ as there may be different cultural and contextual factors which influence the development of these skills in South African children and thus affecting their performance on these tests.

One factor influencing test performance is language. The standardised tests developed in Western countries have English verbal instructions, which are standardised and must be used verbatim ${ }^{3}$. In South Africa, English is only one of I I official languages, thus creating a need to translate instructions when assessing young children. However very little published evidence could be found to support the successful translation of these tests into languages other than English and whether these tools remain reliable when used with populations other than those on which they were standardised.

There has thus been a growing realisation that tests specific to the country and its cultural groups need to be developed or that existing tests need to be adapted to match the needs of the county's population ${ }^{3}$.

\section{STATEMENT OF THE PROBLEM}

In South Africa there is a need for reliable and valid standardised tests that can be translated into languages other than English. One of the tests used to assess perceptual skills in children is the 
Motor-Free Visual Perceptual Test (MVPT-3) ${ }^{8}$. Published data on the MVPT-3 show that this test has acceptable internal consistency and temporal stability during the standardisation process. Content, criteria-related and construct validity were also established. However, it is not clear whether the test remains valid and reliable when translated into languages other than English.

\section{AIM OF STUDY}

The aim of this study was to determine if item bias existed by determining the internal consistency of the items of the Motor-Free Visual Perceptual Test $3^{\text {rd }}$ Edition (MPVT-3) when instructions were translated into Afrikaans and the test was administered to first language Afrikaans speaking children aged 8 years.

\section{LITERATURE REVIEW}

This literature review will examine the use of standardised assessment tools in multicultural, multi-lingual environments, the importance of understanding reliability and validity in these contexts and the influence of language and translation on reliability. The MVPT-3 will be discussed as a test of visual perception used frequently within the South African context.

Assessment in a multicultural, multilingual environment can be challenging, especially when the tools used for assessment were developed on different populations than the one being assessed ${ }^{9}$. In order for the occupational therapist in such an environment to be able to use test results to guide treatment, she must know that the standardised tests are reliable and that the responses to the items on the test that are conceptually related provide consistent results for a given group of respondents and indicate stability. When tests are used with populations for which they have not been designed, test bias can result in a threat to the reliability and the validity of the measures and result in an inaccurate reflection of the child's true ability, especially if the bias occurs due to cultural or language differences ${ }^{10}$. Essential concepts in the application of standardised tests in a multicultural population are therefore bias and equivalence" ${ }^{\prime \prime}$

Equivalence deals with the score comparability across different cultures and is focused on the validity of the test's constructs across different cultural groups as well as differences in different groups' abilities ${ }^{4}$.

Bias, however, refers to systematic sources of variation within test scores that are not as a result of the ability being measured ${ }^{4}$. Bias therefore has to do with the test and three types of bias have been described by van de Vijver and Leung ${ }^{12}$, namely construct bias, method bias and item bias. If the construct being measured differs across cultures then construct bias can occur when the standardised test or measure is used. When a study which determines the suitability of a standardised test or measure for different cultures has faults in the method, (such as the incomparability of samples, instrument characteristics or problems with administration of the test) then method bias occurs. Item bias may be introduced when a test is translated into another language or adapted in another way to be used with children from a population group different to that for which it was designed ${ }^{9}$. This type of bias is most likely to be introduced when tests from other countries, such as the USA or the UK, are introduced to the South African population.

Test reliability related to adaptation or and translation should be of particular concern in countries that are multicultural and multilingual such as South Africa. It is important to state that differences in results found on the tests that have been translated are not due to item bias. This bias may occur as a result of changed meaning as it must ensure the conceptual equivalence of the English on the original version of the measure ${ }^{13}$. When translating tests, it has been shown to be difficult ${ }^{14}$ to ascertain if the observed differences in respondents who speak different languages are due to bias or are real, which can affect the interpretation of the test results ${ }^{15}$.

Dysfunction in visual perception is commonly assessed by occupational therapists and in conjunction with poor motor skills appear to be characteristic of most children with learning problems ${ }^{16}$. The
MVPT-3 is used to assess visual perceptual skills and requires no motor input, but reflects the understanding that perception and cognition go hand in hand and so do not provide different scores for the different processes of perception but rather results in a general score ${ }^{8}$.

The MVPT is one of the most common tests used by American occupational therapists in paediatric practice ${ }^{17}$ and is also widely used in South Africa as a screening test. It was developed and standardised in the United States of America in English. The MVPT-3 test consisted of 65 items, with variable starting and ending points for children of different ages. For children between the ages of $4-10$ years only the first 40 items are administered ${ }^{8}$. Each item is presented in a multiple-choice answer format, with the correct answer selected from four choices arranged horizontally across the page. The norms of the MVPT-3 were derived from a sample of 1856 American individuals from both the urban $(76.8 \%)$ and rural $(23.3 \%)$ areas who were children aged from 4 years to adults 95 years old. The sample was made up of all ethnic groups in the American society of whom $5 \mathrm{I} .8 \%$ female and $48.2 \%$ male and $\mathrm{I} 4 \mathrm{I}$ children were tested in 8-year-old age group.

The authors of the MVPT-3 examined the reliability of the test by evaluating its internal consistency and temporal stability. They concluded that the MVPT-3 could be used with confidence with individuals aged 5 years and older. Content, criterion-related and construct validity were determined for the test and it was concluded that the MVPT-3 adequately represented visual perception, that it was comparable to other available visual perception tests and that it tested visual perception as designed, thus making the MVPT-3 a valid instrument. No studies on culture and gender differences on the MVPT-3 have been published. Very little has been published on the reliability and validity of the test outside of the test authors' own work. However, the work done by Brown and his colleagues ${ }^{18,19}$ with adults has highlighted the need to further interrogate reliability and validity of the tool and has called into question the uni-dimensionality (measurement of one construct) of the test. Further research in different populations is needed in order to fully interrogate the reliability and validity of this tool.

\section{METHOD}

\section{Design}

A descriptive split-half comparative research design was used to determine the internal consistency of the items on the MVPT-3 after the instructions had been translated into Afrikaans.

\section{Sample}

Ethical clearance for this research was obtained from the University of the Witwatersrand Human Research and Ethics Committee and permission from the Department of Education was obtained to carry out this research in Afrikaans-medium primary schools in Pretoria East SA. Children between 8 years 0 months and 8 years II months in grade 2 were selected to participate in this study. These children were selected from nine Afrikaans-medium primary schools in the Pretoria East area. The principal of every second school on the list of schools provided by the Department of Education was approached to participate in the study in order to ensure that all socio-economic areas were represented in the sample. Of the ten schools identified as possible research sites, nine agreed to participate in the study. A total of 80 learners were selected from the nine schools with every fifth child from those whose parents gave permission for them to participate being included in the study.

Inclusion criteria were that the child should be between the ages of 8 years 0 months to 8 years II months, their first language was Afrikaans and they were in Grade 2. There were no explicit exclusion criteria as the study attempted to obtain a true representative sample thus children with learning problems were not excluded.

\section{Research Procedure}

Permission from the Canadian publisher of the MVPT-3 was obtained to translate the instructions of the test into Afrikaans. A rigor- 
ous translation process was followed to ensure the correctness of the translation. A qualified translator translated the test instructions for each plate from English into Afrikaans. A second translator backtranslated the instructions from Afrikaans to English and a third translator checked the back-translation with the original English instructions to ensure that there were no differences ${ }^{14}$.

All participants were assessed on the MVPT-3 at their schools in a quiet, distraction-free environment between 08:30 and II:30 in the morning, excluding first break. All assessments were carried out and scored by one researcher to ensure uniformity of procedure. The assessment took on average 15 minutes. Participants' performance on the test was scored on the standardised scoring sheet provided with the test and were coded to ensure confidentiality. Individual items were marked as either correct or incorrect and summed for a total raw score. Individual and total scores were transcribed to an Excel spreadsheet ${ }^{\circledR}$ and imported into the relevant statistical package.

\section{Data Analysis}

Descriptive statistics were used to describe the sample in terms of age and gender. Internal consistency was calculated in two ways. The first used Cronbach's alpha to determine the internal consistency of all the items on the MVPT-3. Split-half reliability was then used to calculate the internal consistency of two halves of the test, by comparing the first 20 items with the second 20 items using Cronbach's Alpha. Secondary analysis according to gender for internal consistency of items was also completed. The Cronbach's alpha for internal consistency to determine item bias on all items on the test was done using the STATA statistical package.

\section{RESULTS}

The final sample consisted of 43 girls $(53.75 \%)$ and 37 boys (46.25\%). The mean age was 8 years 4 months (SD 2.7 months) with $75 \%(60)$ of the sample falling between 8 years 0 months and 8 years 6 months. A total of ten participants (12.5\%) had diagnosed learning problems and one participant had a visual problem that required corrective spectacles. The majority of the sample (98.8\%) were white children with the remainder (1.2\%) coloured children and was representative of the Afrikaans speaking Pretoria east population, but not the general South African population.

The Cronbach's alpha calculated for all the test items of the 8-year-old Afrikaans-speaking children was found to be 0.804, (Table I) which compared favourably with the results of the American 8 -year-old population, which was 0.83 . Items 4 and 35 scored the same for each child tested in the study and so were dropped from further analyses, as they did not contribute to the calculation of the internal consistency.

However this data set was not entirely comparable as the original American and Canadian data as their item analysis had been done using the SYSTAT statistical package. So to eliminate method bias a second analysis using SYSTAT provided a slightly lower Cronbach's alpha of 0.79 for the overall test (Table I).

An unexpected finding of the item analysis that was different to the American analysis was that some of the items in the analysis showed a negative "sign". This results from an individual item score higher than the overall test score. If an item is a positive question, the sign is expected to be positive. All the instructions in the MVPT3 are positive as learners are asked to identify one shape identical items
Table I: Item analysis of the original 40 items of the MVPT-3 using the STATA and SYSTAT statistical packages

\begin{tabular}{|c|c|c|c|c|c|c|c|}
\hline & & \multicolumn{2}{|c|}{ Cronbach's alpha } & & & \multicolumn{2}{c|}{ Cronbach's alpha } \\
\hline Item & & STATA & SYSTAT & Item & & STATA & SYSTAT \\
\hline & Sign & Alpha & Alpha & & Sign & Alpha & Alpha \\
\hline I & + & 0.78 & 0.77 & $\mathbf{2 1}$ & - & 0.80 & 0.79 \\
\hline $\mathbf{2}$ & + & 0.78 & 0.76 & $\mathbf{2 2}$ & + & 0.78 & 0.79 \\
\hline $\mathbf{3}$ & + & 0.78 & 0.77 & $\mathbf{2 3}$ & + & 0.78 & 0.78 \\
\hline $\mathbf{5}$ & + & 0.80 & 0.78 & $\mathbf{2 4}$ & + & 0.80 & 0.79 \\
\hline $\mathbf{6}$ & + & 0.79 & 0.78 & $\mathbf{2 5}$ & + & 0.80 & 0.79 \\
\hline $\mathbf{7}$ & - & 0.80 & 0.79 & $\mathbf{2 6}$ & + & 0.80 & 0.79 \\
\hline $\mathbf{8}$ & + & 0.79 & 0.78 & $\mathbf{2 7}$ & - & 0.80 & 0.79 \\
\hline $\mathbf{9}$ & + & 0.79 & 0.77 & $\mathbf{2 8}$ & - & 0.81 & 0.79 \\
\hline $\mathbf{I 0}$ & + & 0.78 & 0.76 & $\mathbf{2 9}$ & + & 0.80 & 0.78 \\
\hline $\mathbf{I I}$ & + & 0.80 & 0.78 & $\mathbf{3 0}$ & + & 0.80 & 0.78 \\
\hline $\mathbf{I 2}$ & + & 0.79 & 0.78 & $\mathbf{3 1}$ & + & 0.80 & 0.78 \\
\hline I3 & + & 0.80 & 0.78 & $\mathbf{3 2}$ & - & 0.80 & 0.79 \\
\hline I4 & + & 0.78 & 0.76 & $\mathbf{3 3}$ & - & 0.80 & 0.79 \\
\hline I5 & + & 0.80 & 0.79 & $\mathbf{3 4}$ & - & 0.80 & 0.79 \\
\hline I6 & + & 0.79 & 0.78 & $\mathbf{3 6}$ & + & 0.79 & 0.78 \\
\hline I7 & - & 0.80 & 0.79 & $\mathbf{3 7}$ & + & 0.80 & 0.79 \\
\hline I8 & + & 0.79 & 0.78 & $\mathbf{3 8}$ & + & 0.80 & 0.79 \\
\hline I9 & + & 0.80 & 0.78 & $\mathbf{3 9}$ & + & 0.80 & 0.78 \\
\hline $\mathbf{2 0}$ & + & 0.80 & 0.79 & $\mathbf{4 0}$ & + & 0.80 & 0.79 \\
\hline Test scale & $\mathbf{0 . 8 0}$ & $\mathbf{0 . 7 9}$ & & & & \\
\hline i4 i35 constant in analysis sample, dropped from analysis & & \\
\hline
\end{tabular}

Table II: Different processes of visual perception found in the negative

\begin{tabular}{|c|c|c|c|c|c|c|}
\hline Item & Memory & Discrimination & $\begin{array}{c}\text { Form } \\
\text { Constancy }\end{array}$ & $\begin{array}{c}\text { Figure } \\
\text { ground }\end{array}$ & $\begin{array}{c}\text { Spatial } \\
\text { relations }\end{array}$ & $\begin{array}{c}\text { Visual } \\
\text { Closure }\end{array}$ \\
\hline 7 & & $*$ & $*$ & $*$ & $*$ & $*$ \\
\hline 17 & $*$ & $*$ & $*$ & & $*$ & \\
\hline 21 & $*$ & & $*$ & & $*$ & \\
\hline 27 & $*$ & $*$ & $*$ & & & $*$ \\
\hline 28 & & $*$ & $*$ & & & $*$ \\
\hline 32 & & & $*$ & & $*$ & $*$ \\
\hline 33 & & & $*$ & & $*$ & $*$ \\
\hline 34 & & & $*$ & & $*$ & $*$ \\
\hline Total & $\mathbf{2}$ & $\mathbf{4}$ & $\mathbf{8}$ & $\mathbf{1}$ & $\mathbf{6}$ & $\mathbf{6}$ \\
\hline
\end{tabular}

to that at the top of the page or on the next page. Items 7, 17, 2I, $27,28,32,33$ and 34 all have a negative sign, suggesting some item bias that can be interpreted in two ways. Firstly it could mean that participants interpreted the instructions or the item differently to that intended (Table $I$ ) or that the test does not form a single scale and is not uni-dimensional for this sample of participants ${ }^{20}$.

The eight items which had a negative sign, all contained an element of visual constancy, with two items having a memory element, four a distinct discrimination element, one an element of figure ground, six an element of spatial orientation and seven had an element of visual closure (Table II).

To further investigate the reliability of the test, the split-half reliability was calculated using SYSTAT. The first half of the test (items I - 20) showed more internal consistency than the second half of the test (items 21-40). The Cronbach alpha for the first half of the test was 0.790 and fell within the acceptable range, while the second half of the test was 0.549 , which is an unacceptable level ${ }^{21}$.

Secondary analysis was done to explore whether differences in internal consistency could be detected between genders. Thus the 
Table III: Item analysis of the MVPT-3 raw scores for Gender

\begin{tabular}{|c|c|c|c|c|c|c|c|c|c|c|c|}
\hline \multicolumn{6}{|c|}{ Boys } & \multicolumn{6}{|c|}{ Girls } \\
\hline Item & Sign & Alpha & Item & Sign & Alpha & Item & Sign & Alpha & Item & Sign & Alpha \\
\hline $\mathbf{I}$ & + & 0.64 & 21 & - & 0.679 & I & + & 0.85 & 21 & - & 0.87 \\
\hline 2 & + & & 22 & & & 2 & + & 0.85 & 22 & + & 0.85 \\
\hline 3 & + & 0.67 & 23 & - & 0.67 & 3 & + & 0.85 & 23 & + & 0.85 \\
\hline 4 & & & 24 & - & 0.63 & 4 & & & 24 & + & 0.87 \\
\hline 5 & + & 0.63 & 25 & $=$ & 0.66 & 5 & + & 0.87 & 25 & + & 0.86 \\
\hline 6 & - & 0.66 & 26 & + & 0.64 & 6 & + & 0.86 & 26 & & \\
\hline 7 & - & 0.67 & 27 & + & 0.66 & 7 & - & 0.86 & 27 & - & 0.86 \\
\hline 8 & - & 0.67 & 28 & + & 0.66 & 8 & + & 0.85 & 28 & - & 0.87 \\
\hline 9 & - & 0.66 & 29 & + & 0.65 & 9 & + & 0.85 & 29 & + & 0.86 \\
\hline 10 & & & 30 & + & 0.61 & 10 & + & 0.85 & 30 & + & 0.86 \\
\hline II & + & 0.64 & 31 & + & 0.65 & II & + & 0.86 & 31 & + & 0.86 \\
\hline 12 & + & 0.62 & 32 & + & 0.65 & 12 & + & 0.86 & 32 & - & 0.86 \\
\hline 13 & + & 0.65 & 33 & + & 0.65 & 13 & + & 0.86 & 33 & - & 0.86 \\
\hline 14 & & & 34 & - & 0.67 & 14 & + & 0.85 & 34 & - & 0.86 \\
\hline 15 & - & 0.66 & 35 & & & 15 & + & 0.86 & 35 & & \\
\hline 16 & + & 0.67 & 36 & + & 0.65 & 16 & + & 0.86 & 36 & + & 0.86 \\
\hline 17 & - & 0.67 & 37 & + & 0.64 & 17 & - & 0.86 & 37 & + & \\
\hline 18 & - & 0.65 & 38 & + & 0.64 & 18 & + & 0.86 & 38 & + & 0.87 \\
\hline 19 & - & 0.67 & 39 & + & 0.66 & 19 & + & 0.86 & 39 & + & 0.86 \\
\hline 20 & + & 0.66 & 40 & - & 0.67 & 20 & + & 0.86 & 40 & + & 0.87 \\
\hline \multicolumn{2}{|c|}{ Test Scale } & 0.667 & & & & \multicolumn{2}{|c|}{ Test Scale } & 0.86 & & & \\
\hline \multicolumn{6}{|c|}{$\begin{array}{l}\text { i2, i4, il } 0 \text {, il } 4 \text {, i22, q } 35 \text { constant in analysis sample, } \\
\text { dropped from analysis }\end{array}$} & \multicolumn{6}{|c|}{$\mathrm{i} 4, \mathrm{i} 26, \mathrm{i} 35, \mathrm{i} 37$ constant in analysis sample, dropped from analysis } \\
\hline
\end{tabular}

Table IV: The Cronbach's alpha scores of the first and second halves in the item analysis of the MVPT-3 comparing gender

\begin{tabular}{|l|c|c|}
\hline & Boys & Girls \\
\hline First half (Items I-20) & 0.52 & 0.86 \\
\hline $\begin{array}{l}\text { Second half (Items } \\
\text { 21-40) }\end{array}$ & 0.63 & 0.64 \\
\hline
\end{tabular}

overall internal consistency as well as the split-half reliability was calculated according to gender (Table III). There were significant differences between these two groups ( $\leq \leq 0.000 \mathrm{I})$.

The overall Cronbach's Alpha for the boys was 0.666 while for the girls, it was 0.867 . Thus for the girls the alpha was at an acceptable level while for the boys it was not, suggesting that the MVPT-3 is more internally consistent for the Afrikaans 8-year-old girls than for the Afrikaans 8-year-old boys.

When the sample was split into different gender groups, it emerged that different items needed to be removed from the sample for the different gender groups. In the analysis of the boys' scores, 13 non-contributing items were removed from the analysis (items 2- 4, 7, 8, 10, 14, 17, 19, 21-23, 35 mostly from the first half of the test), to achieve a final Cronbach's alpha of 0.72 , which is just above the acceptable level. In the analysis of girls' scores, seven non-contributing items were removed from the analysis (items 4 , $5,26,28,35,37,40$ ) to achieve a final Cronbach's alpha of 0.88 .

It is interesting to note that when compared to the original STATA analysis in step I there were eight items with negative signs. However, in the item analysis for the boys there were 13 items with negative signs and for the girls there were 10 (Table IV). This suggests that the groups interpreted the instructions for test items differently, with the girls more closely representing the distribution of negative Cronbach's alpha found in the analysis of the whole group.

Split-half calculations according to gender indicated that the second half of the items again had lower consistency and that this was similar for boys and girls (Table III). The girls' split-half reliability results mirrored the overall sample results with the first half of the test showing more internal consistency than the second half. However, for the boys, the split-half reliability scores were both below acceptable standards for alpha, with the second half score higher than the first half of the test.

\section{DISCUSSION}

This study generated some interesting results. At first glance it would appear that, while translating instructions into Afrikaans seemed to influence the internal consistency of the MVPT-3 negatively, the overall alpha score was still relatively high at 0.79 and within acceptable levels for clinical use ${ }^{21}$. However, with further analysis it became clear that internal consistency and thus reliability of this tool in South Africa is far more complex and warrants more careful consideration.

These were the items which revealed that Cronbach's alphas were higher than the overall test Cronbach's alpha. Unfortunately it was impossible to compare the data analyses done on the South African children to that done on the American children as the publisher of the MVPT-3 sent only the analysis done on the age group 4-10, which was not helpful in comparing the different Cronbach's alpha scores within the 8-year-old age band. This raises concern about the reliability of the test as specific scores for each age band are not available even for the American child and there are marked differences in the abilities of a 4 and 8-year-old in terms of visual perception. 
There were also some homogeneous items that were either all correctly or incorrectly answered by all the participants suggesting that the questions were either too easy or too difficult. During the overall analysis items 4 and 35 were answered correctly by all the participants so they did not contribute to the overall testing and reliability of the test. Item 4 falls into the section that Colarusso and Hammill consider being visual discrimination and item 35 falls into the spatial orientation. These two items were not able to differentiate visual perception skills in this 8 year old sample 8 .

On evaluation it was noted that most of the negative noncontributing items were in the second half of the test. This was confirmed when a split-half evaluation was done, as it was found that the first half of the test had a higher Cronbach's alpha score than the second half. The negative Cronbach's alphas are difficult to explain ${ }^{20}$. A number of items use the same instructions and although the negative items were grouped other items with the same translated instructions did not have a negative sign. It is therefore possible that it was not misinterpretation due to translation which resulted in item bias but the differences in the ability of the visual perceptual abilities of the Afrikaans speaking participants. These results indicate possible cultural differences in these participants' ability to interpret the test items which assess visual form constancy, spatial relations and visual closure.

Although the authors of the MVPT-3 have claimed uni-dimensionality (measuring one construct) for the MVPT-3, this has been called into question in a study that looked at factor analysis when using the test with adults ${ }^{20}$. This study found that the MVPT-3 showed characteristics of multi-dimensionality (thus measuring more than one construct) and thus urged caution in interpreting the MVPT-3 as an appropriate overall measure of visual perception in adults ${ }^{20}$. The findings of negative signs in this study may also support this finding of multi-dimensionality and certainly warrants further investigation.

To further confirm these differences the results from the boys and girls were considered as they also showed differences. Gender differences in the development of visual perceptual skills have been reported in the literature ${ }^{22,23,24}$. It is thus interesting to note that the differences noted between the performance of Afrikaansspeaking girls and boys seem to reflect some of these reported developmental differences. Girls showed much more variability of performance in the second half of the test, which includes predominantly visual spatial tasks and manipulating images within their minds ${ }^{25}$. This variability affected the split-half reliability and gave a reliability score below acceptable levels for the second half of the test for girls. However, there was much less variability in girls' performance whose tasks were predominantly focussed on form recognition and memory (including figure-ground, and constancy) and the first half of the test generated good reliability scores. This appears to reflect developmental trends that have shown visual spatial perception to develop later in girls ${ }^{26}$.

The trend was reversed in the boys' performance. Although overall the boys' performance on the MVPT-3 was much more variable than the girls' resulting in below acceptable reliability rates, it is still interesting to note that reliability on the second half of the test was better than the first. This suggests that variability on spatial perceptual tasks was less than variability on object recognition and memory tasks, again reflecting the trends in development reported in literature. However, both the split-half reliability scores and the overall reliability scores suggest that the manner in which perceptual tasks are presented to boys in this test do not reliably test those concepts and results should be treated with caution.

In the analysis for gender differences items that were homogeneous for each group were items 2, 4, 10, 22 and 35 for the boys, and items 4, 26, 35 and 37 for the girls. With further consideration it is seen that the boys have more questions that are homogeneous (answered correctly by the total sample) than the girls and that the boys' homogenous questions tend to be in the first half of the test, i.e. in the visual discrimination, form constancy, visual closure and spatial orientation sections of the test whereas the girls' are in the visual discrimination, visual closure and spatial orientation sections of the test. Most of the questions for which the boys had homogeneous scores there is a strong form constancy and spatial orientation element, whereas the spatial element is not as obvious in the homogenous items identified for the girls. All the items that were homogeneous for the girls had a strong form constancy element. This trend supports the findings that girls and boys develop visual perception differently and at a different rate ${ }^{26}$. Thus the use of one set of norms to measure visual perceptual skills of boys and girls together is questionable and care must be taken in interpreting these scores, especially those of boys.

\section{Limitations of the study}

The study sample of $98.9 \%$ white children and I.2\% coloured children was representative of the Afrikaans speaking Pretoria east population but this was not representative of the population of South Africa so results cannot be generalised to other areas in South Africa.

Eighty eight percent of the participants fell in the age range of 8 years 0 months to 8 years 7 months due to the inclusion criteria that they be in Grade 2, which means that there were very few children closer to the age of 9 years in the sample.

As the results of this study were not compared to an Englishspeaking South African population, it is difficult to say with certainty that the translation of instructions influenced the internal consistency of the MVPT-3 or whether the South African population shows different trends to the standardised population. Research on the equivalence of visual perceptual tests need to be carried out on South African children to determine the effects if any of different cultures on their ability in this area.

\section{CONCLUSION}

The results of this study highlight an important question. Is it sufficient for a clinician to only look at overall reliability and validity when considering the use of an imported standardised tool? Unfortunately the results of this study could not be directly compared to the results of the original standardisation population as the publisher did not release these data to the researchers. This makes it difficult to decide whether the results obtained in this study are completely due to the translation of instructions, whether South African populations display different developmental trends, or whether the gender differences are universal. These results highlight the deficits in reporting only overall reliability and validity for any tool. It is a general trend in the reporting of standardisation processes in manuals to only report on overall scores. It is also common for tool developers to underplay below acceptable reliability scores in subtests, or subdivided groups as long as the final, overall reliability scores are above 0.75 . Thus it is necessary for clinicians to exercise caution when using any imported standardised assessment tool and to recognise that the reliability of that tool may be much more complex than at first meets the eye.

\section{Recommendations}

From this study it is clear that that internal consistency and reliability is complex and needs to be carefully interrogated within the South African populations. South African occupational therapists need to be aware of the uses and limitations of standardised assessment tools and interpret results with care. Firstly occupational therapists need to be aware that the translation of tests can change the meaning of even very simple instructions and that the reliability of the test may be influenced creating bias and thus inaccurate test results. Secondly culture and experience play a role in normal development and thus, even though the developers of a test may claim the test to be culturally "neutral", the development of the skills tested may differ in the South African populations. Thirdly occupational therapists should be aware of gender differences and developmental differences and, where possible, compare a child's performance to gender norms. 
In conclusion, standardised assessment tools have the appearance of being a respectable way to assess skills. In a multi-cultural, multi-lingual environment, the challenges of using an imported standardised assessment cannot be ignored. Occupational therapists may be better off using their clinical reasoning, activity analysis and observational skills to overcome these challenges. This may ensure more accurate and holistic assessments in the South African context.

\section{REFERENCES}

I. Richmond J, Holland K. Correlating the Developmental Test of Visual Perception-2 (DTVP-2) and the Test of Visual Perceptual Skills Revised (TVPS-R) as assessment tools for learners with learning difficulties. South African Journal of Occupational Therapy, 20II; 4I(I): 33-37.

2. Knowles J. Assessment of non-verbal intelligence in South African Schools: Do language and gender bias performance on the Raven's Standard Progressive Matrices? MSc Research report. Johannesburg: University of the Witwatersrand; 2008.

3. Alers V, Crouch R. Occupational Therapy: An African Perspective. Johannesburg: Shakespeare; 20II.

4. Van de Vijver F, Tanzer NK. Bias and equivalence in cross-cultural assessment: An overview. European Review of Applied Psychology. 1997; 47(2).

5. Thorley M, Lim SM. Considerations for occupational therapy assessment for indigenous children in Australia. Australian Occupational Therapy Journal. 20I I; 58(I): 3-I0.

6. Josman N, Abdallah TM, Engel-Yeger B. Cultural factors affecting the differential performance of Israeli and Palestinian children on the Loewenstein Occupational Therapy Cognitive Assessment. Research in Developmental Disabilities. 2010; 3I(3): 656-663.

7. Rosselli M, Ardila A. The impact of culture and education on nonverbal neuropsychological measurements: a critical review. Brain and Cognition. 2003; 52(3): 326-333.

8. Colarusso R, Hammill D. Motor-Free Visual Perceptual Test, Third Edition Novato: Academic Therapy Publications; 2003.

9. Kristjansson E, Desrochers A, Zumbo B. Translating and Adapting Measurement Instruments for Cross-Linguistic and Cross-Cultural Research: A Guide for Practitioners. Canadian Journal of Nursing Research. 2003; 35(2): 127-142.

10. Camilli G, Shepard L. Methods for identifying biased test items. London: Sage; 1994.

II. Meiring D, van de Vijver A, Rothman S, Barrick M. Construct, item, and method bias of cognitive and personality tests in South Africa. SA Journal of Industrial Psychology. 2005; 3 I (I): I-8.

12. Van de Vijver F, Leung K. Methods and data analysis for cross-cultural research. Thousand Oaks, California: Sage Publications, Inc; 1997.

13. Hambleton R. Adapting achievement tests into multiple languages for international assessments. In Porter A, Gamoran A. (Eds.), Methodological advances in cross-national surveys of educational achievement. Washington: National Academy Press; 2002.

14. Banville D, Desrosiers P, Genet-Volet Y. Translating questionnaires and inventories using a cross-cultural translation technique. Journal of Teaching in Physical Education. 2000; 19(3): 374-387.

I5. Sirecci $S$. Problems and issues in linking assessments across cultures. Educational Measurement: Issues and Practices. 1997; I6(I): I2-19.

16. Murray E, Cermak S, O'Brien V. The Relationship between Form and Space perception, Constructional Abilities, and Clumsiness in Children. American Journal of Occupational Therapy. 1990; 44(7): 623-628.

17. Burtner P, McMain M, Crowe T. Survey of Occupational Therapy Practitioners in Southwestern Schools: Assessments Used and Preparation of Students for School-Based Practice. Physical \& Occupational Therapy in Pediatrics. 2002; 22(I):25-39.

18. Brown T, Elliot S. Factor Structure of the Motor-Free Visual Perception Test-3rd edition (MVPT-3). Canadian Journal of Occupational Therapy. 201 I; 78(I):26-36.

19. Brown T. An examination of the construct validity of the Motor-Free Visual Perceptual Test-Third Edition (MVPT-3) using Rasch analysis with adult participants. OTJR: Occupation, Participation \& Health. 20II; 3I(2): 73-80.

20. Nichols D. My Coefficient is Negative! [Online]; 1999 [cited 2013 October 23. Available from: http://www.ats.ucla.edu/stat/spss/ library/negalpha.htm.
21. Peterson R. A Meta-Analysis of Cronbach's Coefficient Alpha. Journal of Consumer Research. 1994; 2 I (2): 38I-39I.

22. Atkinson J, Nardini M. The Neuropsychology of Visuospatial and Visuomotor Development. In Reed J, Warner-Rogers J. Child Neuropsychology: Concepts, Theory, and Practice. Chichester: Wiley-Blackwell; 2008.

23. Krinzinger $\mathrm{H}$, Wood $\mathrm{G}$, Willmes $\mathrm{K}$. What accounts for individual and gender differences in the multi-digit number processing of primary school children? Zeitschrif fur Psychologie. 20I2; 220(2): 78-89.

24. Lange-Kuttner C, Ebersbach M. Girls in detail, boys in shape: Gender differences when drawing cubes in depth. British Journal of Psychology. 2012; 104(3):4I3-437.

25. Mao HF, Li W, Lo JL. Construction validity of Beery's developmental test of visual motor integration for Taiwanese children. OTJR: Occupation, Participation and Health. 1999; 19(4): 24I-257.

26. Snell D. Sex Differences in Cognitive and Personal - Social Development of Young Children. Research Dissertation. University of the Witwatersrand; 1982.

27. Isaac KM. Speech Pathology in Cultural and Linguistic Diversity. London: Whurr; 2002.

28. Stow $C$, Dodd B. Providing an equitable service to bilingual children in the UK: a review. International Journal of Language and Communication Disorders. 2003; 38(4): 35I-377.

29. Van de Vijver F. Cross-cultural assessment: Value for money? Applied Psychology: An International Review. 2002; 5 I (4): 545-566.

\section{Corresponding author}

\section{Lyndsay Koch}

Department of Occupational Therapy, School of Therapeutic Sciences, Faculty of Health Sciences, University of the Witwatersrand, York Rd Parktown, Johannesburg, South Africa. Lyndsay.Koch@wits.ac.za 